\title{
Preoperative volumetric synthetic magnetic resonance imaging of the primary tumor for a more accurate prediction of lymph node metastasis in rectal cancer
}

\author{
Li Zhao ${ }^{1}$, Meng Liang ${ }^{1}$, Zhuo Shi $^{1}$, Lizhi Xie ${ }^{2}$, Hongmei Zhang ${ }^{1}$, Xinming Zhao ${ }^{1}$ \\ ${ }^{1}$ Department of Diagnostic Radiology, National Cancer Center/National Clinical Research Center for Cancer/Cancer Hospital, Chinese Academy of \\ Medical Sciences and Peking Union Medical College, Beijing, China; ${ }^{2}$ GE Healthcare, Magnetic Resonance Research China, Beijing, China \\ Correspondence to: Hongmei Zhang; Xinming Zhao. Department of Diagnostic Radiology, National Cancer Center/National Clinical Research Center \\ for Cancer/Cancer Hospital, Chinese Academy of Medical Sciences and Peking Union Medical College, 17 Panjiayuan Nanli, Chaoyang District, \\ Beijing 100021, China. Email: zhanghongmei1973@163.com; zhaoxinming@cicams.ac.cn.
}

Background: An accurate assessment of lymph node (LN) status in patients with rectal cancer is important for treatment planning and an essential factor for predicting local recurrence and overall survival. In this study, we explored the potential value of histogram parameters of synthetic magnetic resonance imaging (SyMRI) in predicting LN metastasis in rectal cancer and compared their predictive performance with traditional morphological characteristics and chemical shift effect (CSE).

Methods: A total of 70 patients with pathologically proven rectal adenocarcinoma who received direct surgical resection were enrolled in this prospective study. Preoperative rectal MRI, including SyMRI, were performed, and morphological characteristics and CSE of LN were assessed. Histogram parameters were extracted on a T1 map, T2 map, and proton density (PD) map, including mean, variance, maximum, minimum, 10th percentile, median, 90th percentile, energy, kurtosis, entropy, and skewness. Receiver operating characteristic (ROC) curves were used to explore their predictive performance for assessing LN status.

Results: Significant differences in the energy of the T1, T2, and PD maps were observed between LNnegative and $\mathrm{LN}$-positive groups [all $\mathrm{P}<0.001$; the area under the ROC curve (AUC) was 0.838, 0.858, and 0.823 , respectively]. The maximum and kurtosis of the $\mathrm{T} 2$ map, maximum, and variance of $\mathrm{PD}$ map could also predict $\mathrm{LN}$ metastasis with moderate diagnostic power ( $\mathrm{P}=0.032,0.045,0.016$, and 0.047 , respectively). Energy of the T1 map [odds ratio $(\mathrm{OR})=1.683$, 95\% confidence interval (CI): 1.207-2.346, $\mathrm{P}=0.002$ ] and extramural venous invasion on MRI (mrEMVI) (OR =10.853, 95\% CI: 2.339-50.364, $\mathrm{P}=0.002$ ) were significant predictors of LN metastasis. Moreover, the T1 map energy significantly improved the predictive performance compared to morphological features and CSE $(\mathrm{P}=0.0002$ and 0.0485$)$.

Conclusions: The histogram parameters derived from SyMRI of the primary tumor were associated with LN metastasis in rectal cancer and could significantly improve the predictive performance compared with morphological features and CSE.

Keywords: Lymphatic metastasis; magnetic resonance imaging (MRI); rectal neoplasms

Submitted May 14, 2020. Accepted for publication Nov 22, 2020.

doi: 10.21037/qims-20-659

View this article at: http://dx.doi.org/10.21037/qims-20-659 


\section{Introduction}

Lymph node (LN) metastasis is an essential prognostic factor for overall survival (OS) in rectal cancer (1). Treatment strategies for rectal cancer patients are based on the risk stratification for local recurrence and distant metastasis, in which tumor, node, metastasis (TNM) staging is the main factor (2). Total mesorectal excision is usually considered for low-risk patients, while preoperative neoadjuvant chemoradiotherapy (nCRT) is recommended for highrisk patients with locally advanced rectal cancer (2-4). Therefore, an accurate preoperative LN stage is crucial for treatment planning.

Magnetic resonance imaging (MRI) is the accepted standard method for preoperative local staging of rectal cancer (4). The most common criteria are the traditional morphological findings like short-axis diameter, shape, border, and signal intensity (5). However, these characteristics' diagnostic accuracy and interobserver agreement remain challenging for evaluating $\mathrm{LN}$ metastasis (6). The chemical shift effect (CSE) at the border of normal LNs is valuable in identifying metastatic LNs; yet, up to $31.2 \%$ of small benign LNs with long-axis diameters $<3 \mathrm{~mm}$ present with an absence of CSE (7). Previous studies have suggested that quantitative functional images could be useful for predicting metastatic LNs (8-11). Some of these studies have used a node-by-node approach to match the LNs on MRI (mrLNs) with the pathological specimen, which currently represents the major obstacle in nodal research (9), while others have focused on the relationships between the characteristics of the primary tumor and the LN (10,11).

Synthetic MRI (SyMRI) is a novel technology that can simultaneously generate multiple sequences in a single scan, including morphological images like T1-weighted imaging (T1WI), T2-weighted imaging (T2WI), and quantitative images of T1 map, T2 map, and proton density (PD) map. There have been some promising findings of SyMRI in brain, bone, articulatio, breast, etc. (12-16). A previous study suggested that the $\mathrm{T} 2$ value derived from a $\mathrm{T} 2 \mathrm{map}$ is valuable for identifying prostate cancer and could reflect the tumor aggressiveness (17). Another study reported that the $\mathrm{T} 2$ value in breast cancer responders was reduced after neoadjuvant chemotherapy (18).

Histogram parameters reflect the distribution of voxel gray-level intensity with good repeatability (19). Previous studies have shown that the primary tumor histogram parameters on T2WI, diffusion-weighted imaging (DWI), or contrast-enhanced MRI were associated with tumor response to nCRT, survival, histological grade, and LN metastasis in rectal cancer (20-23). To our knowledge, studies evaluating the relationship between histogram parameters derived from SyMRI of the primary tumor and regional LN metastasis in rectal cancer are scarce. Therefore, this study aimed to explore the potential value of histogram parameters derived from SyMRI of the primary tumor in predicting regional $\mathrm{LN}$ metastasis in rectal cancer and to compare their predictive performance with traditional morphological characteristics and CSE.

\section{Methods}

\section{Participants}

This prospective study was approved by the Institutional Review Board of the Cancer Hospital, Chinese Academy of Medical Sciences (NCC2019C-040), and informed consent was provided by all participants. A total of 131 patients suspected of having rectal cancer between January 2019 and November 2019 were recruited for this study. Patients with locally advanced rectal cancer usually receive nCRT in our hospital, except for some specific palliative situations. Inclusion criteria were as follows: (I) underwent preoperative rectal MRI examination including SyMRI within 4 weeks of their surgery, (II) without nCRT before the surgery, (III) histopathologically proven rectal adenocarcinoma. A total of 61 patients were excluded for the following reasons: (I) received nCRT before the surgery $(n=38)$, (II) without treatment records at our hospital ( $\mathrm{n}=14)$, (III) with other pathological types such as mucinous adenocarcinoma, neuroendocrine carcinoma, and melanoma ( $\mathrm{n}=5)$, (IV) insufficient imaging quality $(\mathrm{n}=2)$, (V) time interval between MRI and surgery $>4$ weeks $(n=2)$. Consequently, 70 patients were enrolled and allocated to $\mathrm{LN}$-negative (LN-, pN0) and LN-positive (LN+, pN1-2) groups based on the pathological $\mathrm{N}$ stage. The flowchart of participants is shown in Figure 1.

\section{Clinical and pathological characteristics}

Clinical status included gender, age, carcinoembryonic antigen (CEA), and carbohydrate antigen 199 (CA199). CEA and CA199 thresholds were based on the normal ranges at our institution $(0-5 \mathrm{ng} / \mathrm{mL}$ for CEA and $0-37 \mathrm{U} / \mathrm{mL}$ for CA199). The pathological characteristics of surgical specimens were assessed according to the $8^{\text {th }}$ edition of the 


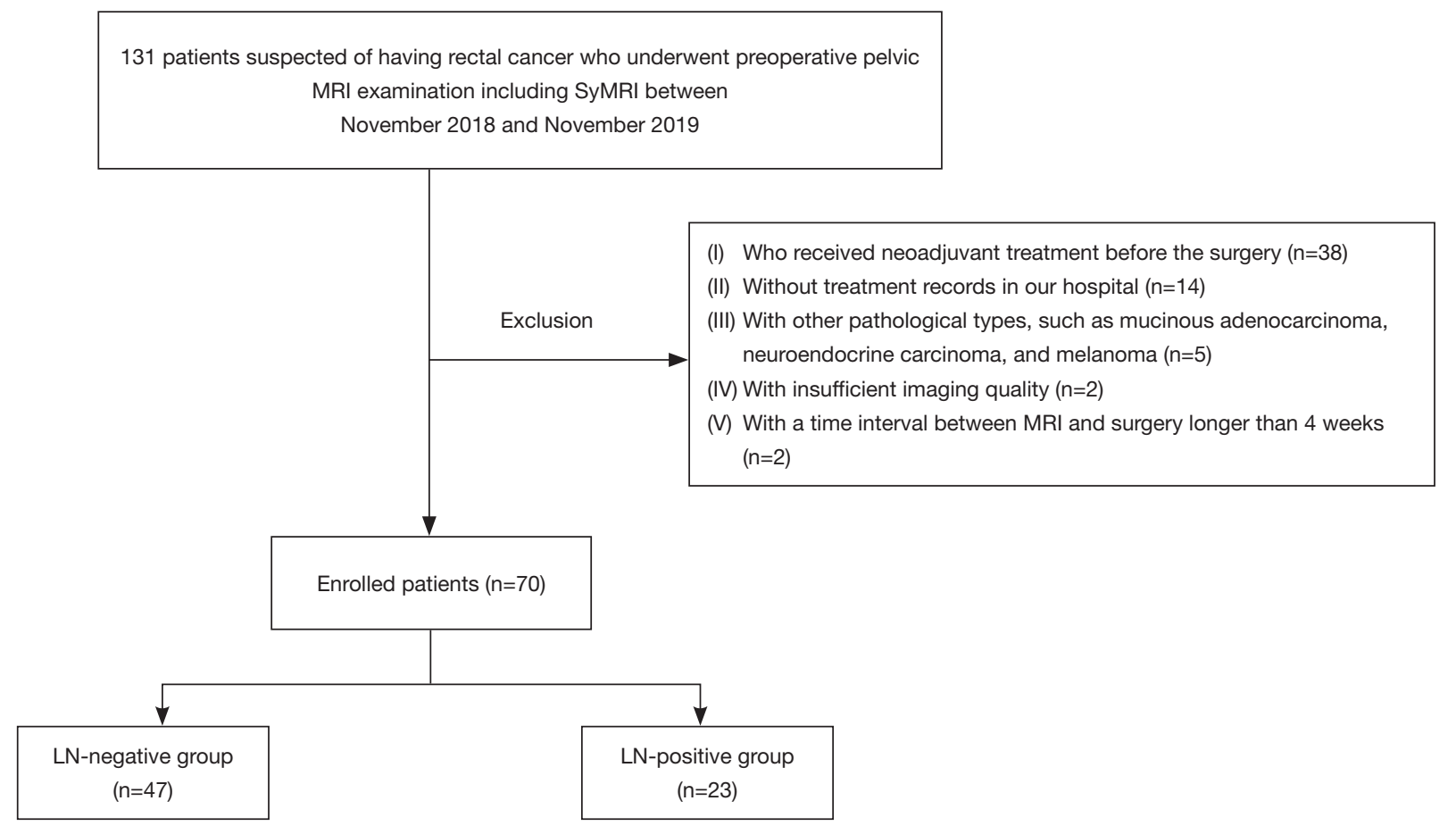

Figure 1 Flowchart of the enrolled patients. SyMRI, synthetic magnetic resonance imaging; LN, lymph node.

American Joint Committee on Cancer (AJCC $8^{\text {th }}$ ) TNM staging system. In addition, the degree of differentiation, pathological $\mathrm{T}$ stage, $\mathrm{N}$ stage, and pathological extramural venous invasion (pEMVI) of the tumor were collected.

\section{MRI protocol}

The MRI was performed on a 3T scanner (Signa Pioneer, GE Healthcare, Milwaukee, WI, USA) using a phased array body coil. Before the examination, two glycerin enemas were routinely used to evacuate the bowels. The participants underwent endorectal filling with ultrasound transmission gel $(60-100 \mathrm{~mL})$ to highlight the tumor borders and antispasmodic medications (except those with contraindications) before conducting the MRI examinations. Consequently, oblique axial (angulated perpendicular to the long axis of the tumor), oblique coronal (angulated parallel to the long axis of the rectum), and sagittal T2weighted spin-echo sequences were acquired according to the following parameters: repetition time, 5,115, 5,912, 5,062; echo time, 85.0, 98.8, 90.0; echo train length, 24, 32, 24; slices, 24; slice thickness, $3.0 \mathrm{~mm}$; spacing, 0.3 ; the field of vision (FOV), $18 \times 18,24 \times 24,24 \times 24 \mathrm{~cm}$; matrix, 320×224, $320 \times 256,320 \times 224$; scanning time, $02: 39,02: 40,02: 37$. For
SyMRI, a multi-dynamic multiecho (MDME) sequence with two echo times $(19.5 / 97.3 \mathrm{msec})$ and four saturation delay times $(210 / 610 / 1,810 / 3,810 \mathrm{msec})$ was used. The acquisition parameters of the oblique axial MDME sequence were as follows: repetition time/echo time, 4,000/19.5 msec; flip angle, $90^{\circ}$; echo train length, 16; slices, 24; slice thickness, $3.0 \mathrm{~mm}$; spacing, 0.3 ; FOV, $24 \times 24 \mathrm{~cm}$; matrix, 320×256; total scanning time, 4 min and $32 \mathrm{~s}$. All sequences were obtained during free breathing.

\section{Imaging analysis}

Two radiologists with 5 and 10 years of experience in rectal imaging, who were blinded to the pathological data, evaluated the MR images, including tumor location, maximum tumor length (MTL), circumferential percentage (CP) of the intestinal lumen, T stage, EMVI, and LN status. All these features were evaluated using T2-weighted spin-echo sequences. Tumor location was defined by the distance from the inferior part of the tumor to the anal verge on sagittal T2WI (lower rectum, $<5 \mathrm{~cm}$; middle rectum, 5-10 cm; and upper rectum, $>10 \mathrm{~cm})$. The MTL was obtained from the slice with the maximum longitudinal length of the tumor on sagittal T2WI. The CP was assessed 

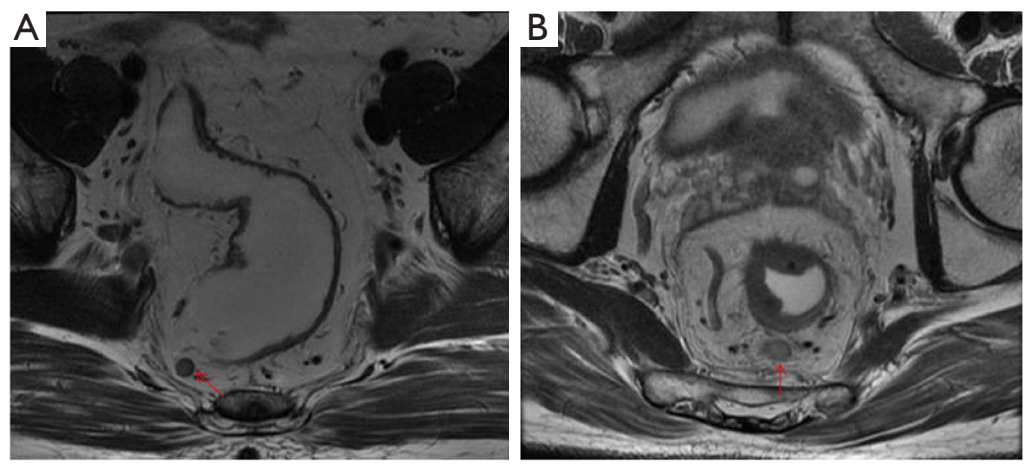

Figure 2 Representative images of CSE. (A) A 54-year-old male with rectal cancer; the arrow showing a LN with smooth and regular CSE on oblique axial T2WI; (B) a 66-year-old male with rectal cancer; the arrow showing a LN with irregular and interrupted CSE on oblique axial T2WI. CSE, chemical shift effect; LN, lymph node; T2WI, T2-weighted imaging.

at the slice showing the largest tumor on oblique axial T2WI according to the percentage of circumferential tumor invasion in the rectal wall and was divided into four groups (group 1, <1/4; group 2, 1/4-1/2; group 3, 1/2-3/4; group $4,>3 / 4)$. The $\mathrm{T}$ stage on MRI (mrT stage) and EVMI on MRI (mrEMVI) were evaluated based on the European Society for Medical Oncology (ESMO) guidelines (2). The LN status was assessed according to the conventional morphological characteristics of short-axis diameter, border, and intensity, and then the CSE was added for an overall evaluation. Specifically, LNs with a short-axis diameter $\geq 5 \mathrm{~mm}$, irregular border, heterogeneous signal intensity, or absence of smooth and regular CSE were identified as positive LN (Figure 2). If there was any obvious discordance between the two radiologists in their assessment of MR features, a senior radiologist with 20 years of rectal imaging experience evaluated it again and made the final decision.

The acquired SyMRI was processed with SyMRI software (version 8.0, SyntheticMR, Linköping, Sweden) to generate the quantitative maps (T1 map, T2 map, and PD map) and T2WI (SyT2WI). Then, SyT2WI was loaded into ITK-SNAP software (version 2.2.0, www.itksnap.org) for segmentation. For each patient, a radiologist (reader 1 , with 5 years of experience in rectal imaging) who was blinded to all the clinical information, manually delineated the whole tumor regions of interest (ROIs) along the border of the primary tumor on each section, excluding obvious cystic and necrotic areas (Figure 3). The ROIs were later copied to the T1 map, T2 map, and PD map. After 2 weeks, 30 patients were randomly selected to segment again by reader 1 and another radiologist (reader 2, with
10 years of experience of rectal imaging) to assess intra-/ inter-reader agreement. Open-source Pyradiomics (http://www.radiomics.io/pyradiomics.html) (24) was used to extract histogram parameters from quantitative maps, including mean, variance, maximum, minimum, $10^{\text {th }}$ percentile, median, $90^{\text {th }}$ percentile, energy, kurtosis, entropy, and skewness.

\section{Statistical analysis}

Group comparison for continuous variables was performed by using the $t$-test or the Mann-Whitney $\mathrm{U}$ test according to the normality of data distribution. Categorical variables were compared using the chi-square test or Fisher exact test. The intraclass correlation coefficient (ICC) of histogram parameters was investigated for the intra-observer and interobserver agreement. Features with ICC values $<0.8$ were excluded. Spearman correlation analysis was performed to evaluate the correlation between the histogram parameters and LN metastasis. Receiver operating characteristic (ROC) curves were applied to assess histogram parameters' predictive performance in LN metastasis. The Youden index determined the optimal threshold, and the area under the ROC curve (AUC), sensitivity, specificity, positive predictive value (PPV), and negative predictive value (NPV) were calculated. Collinearity diagnosis was then performed with a variance inflation factor $(\mathrm{VIF})>10$, indicating the existence of collinearity. Subsequently, multivariate binary logistic regression analysis was conducted with a forward selection of variables to evaluate the performance of distinguishing LN metastasis. Differences in diagnostic efficiency of 

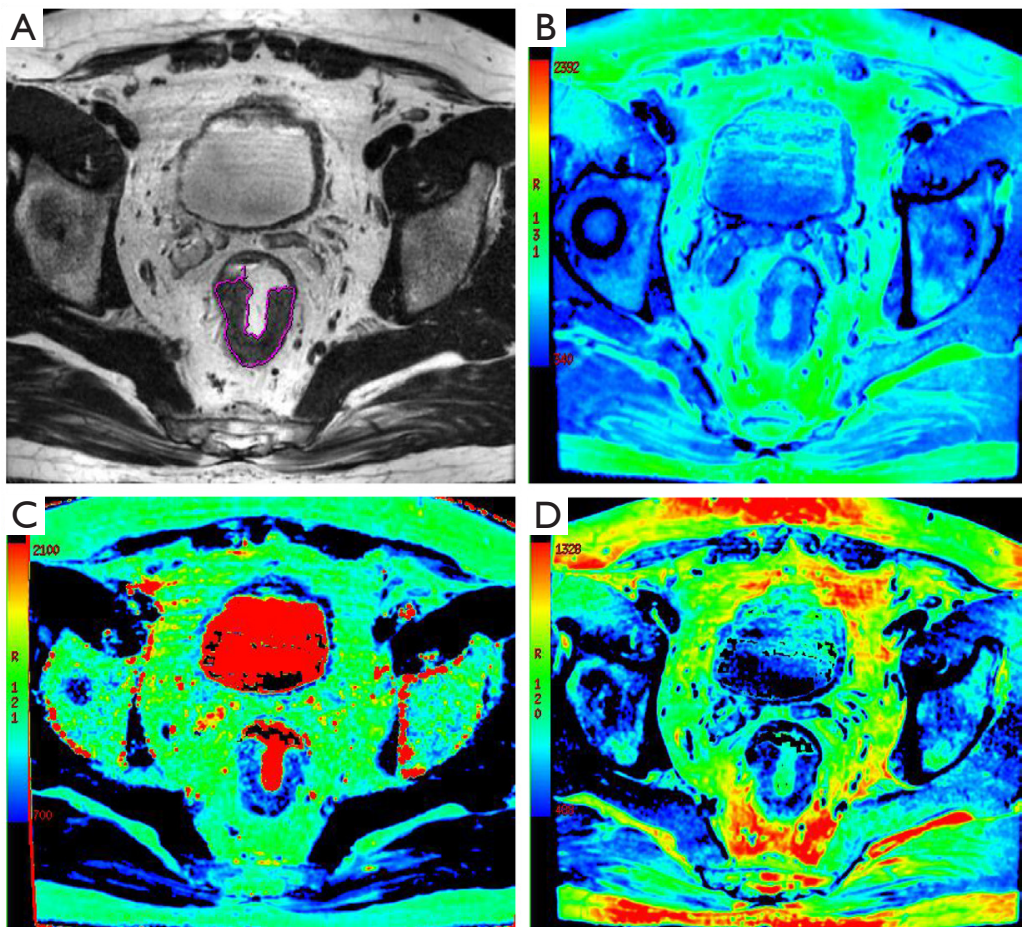

Figure 3 SyMRI of a 49-year-old male patient. (A) ROIs were manually drawn along the border of the tumor on T2WI of SyMRI; (B-D) T1 map, T2 map, and PD map at the same level as figure (A). SyMRI, synthetic magnetic resonance imaging; ROIs, regions of interest; T2WI, T2-weighted imaging.

different methods for predicting LN status were analyzed using the DeLong test. A two-sided $\mathrm{P}$ value $<0.05$ was considered statistically significant. All statistical analyses were performed using SPSS 20.0 (IBM, Armonk, NY, USA) and MedCalc 11.4 (MedCalc, Mariakerke, Belgium).

\section{Results}

\section{Demographic and pathological characteristics}

A total of 70 patients were included in the study. Among those, $48(68.6 \%)$ were male, and $22(31.4 \%)$ were female, with a mean age of 58 [36-76] years old. All patients received radical surgical resection, including abdominoperineal resection (APR) $(\mathrm{n}=21)$, low anterior resection (LAR) or anterior resection (AR) $(n=45)$, Hartman's resection $(n=3)$, and other types of resection $(\mathrm{n}=1)$. The median MTL measured on sagittal T2WI was $3.5(1.5-6.8) \mathrm{cm}$. A total of 4 patients were pathologically reported as pT1; 28 were pT2; 31 were pT3; 7 were pT4. In addition, 47 patients were pathologically confirmed with
pN0, 12 with pN1 (pN1a, 8; pN1b, 4), and 11 with pN2 (pN2a, 4; pN2b, 7); there were 47 patients in $\mathrm{LN}$ - group, and 23 in LN+ group.

The clinical and pathological characteristics in the two groups are summarized in Table 1. The CEA and CA199 levels, pathological T stage, and EMVI were significantly different in the two groups $(\mathrm{P}=0.035,0.035,<0.001$, and 0.003 , respectively); while no difference was found in age, gender, type of surgery, and histologic grade.

\section{Imaging findings}

Radiological features are listed in Table 2. Assessment of MLT, mrT stage, and mrEMVI was proven to be statistically different between the two groups $(\mathrm{P}<0.001)$. In 15 patients, LNs were evaluated as false-positive based on morphological features compared with pathology results and were correctly assessed in terms of CSE.

The histogram parameters with statistically significant differences between the two groups and their ROC curve 
Table 1 Clinical and pathological data of participants

\begin{tabular}{|c|c|c|c|}
\hline Characteristics & $\mathrm{LN}-(\mathrm{n}=47)$ & $L N+(n=23)$ & $P$ value \\
\hline Age (years), mean \pm SD & $58.53 \pm 9.28$ & $56.26 \pm 10.07$ & 0.527 \\
\hline Gender, n (\%) & & & 0.900 \\
\hline Male & $32(68.1)$ & $16(69.6)$ & \\
\hline Female & 15 (31.9) & $7(30.4)$ & \\
\hline CEA level (ng/mL), $\mathrm{n}(\%)$ & & & $0.035^{\star}$ \\
\hline$<5$ & $42(89.4)$ & $15(65.2)$ & \\
\hline$\geq 5$ & $5(10.6)$ & $8(34.8)$ & \\
\hline $\begin{array}{l}\text { CA199 level (U/mL), } n \\
(\%)\end{array}$ & & & $0.035^{\star}$ \\
\hline$<37$ & $45(95.7)$ & $18(78.3)$ & \\
\hline$\geq 37$ & $2(4.3)$ & $5(21.7)$ & \\
\hline Type of surgical, n (\%) & & & 0.076 \\
\hline APR & $18(38.3)$ & $3(13.0)$ & \\
\hline LAR or AR & $26(55.3)$ & $19(82.6)$ & \\
\hline Hartman's resection & $2(4.3)$ & $1(4.3)$ & \\
\hline Others & $1(2.1)$ & $0(0.0)$ & \\
\hline Histologic grade, n (\%) & & & 0.443 \\
\hline Well or moderate & $31(66.0)$ & $13(56.5)$ & \\
\hline Poor & $16(34.0)$ & $10(43.5)$ & \\
\hline pT stage, n (\%) & & & $<0.001^{*}$ \\
\hline $1 / 2$ & $30(63.8)$ & $2(8.7)$ & \\
\hline $3 / 4$ & 17 (36.2) & $21(91.3)$ & \\
\hline pEMVI, n (\%) & & & $0.003^{*}$ \\
\hline Absent & $40(85.1)$ & $12(52.2)$ & \\
\hline Present & 7 (14.9) & $11(47.8)$ & \\
\hline
\end{tabular}

*, $\mathrm{P}<0.05$. LN-, pathological N0 stage; LN+, pathological N1-N2 stage; SD, standard deviation; CEA, carcinoembryonic antigen; CA199, carbohydrate antigen 199; APR, abdominoperineal resection; LAR, low anterior resection; AR, anterior resection; pEMVI, pathological extramural venous invasion; LN, lymph node.

results are shown in Table 3. The ICC values of these parameters were $>0.8$, reflecting excellent inter- and intraobserver agreement. The significantly higher energy of the T1 map, T2 map, and PD map was found in the $\mathrm{LN}+$ compared to the $\mathrm{LN}$ - group $(\mathrm{P}<0.001$; Figure $4 A, B, C)$, and showed significant positive correlations with $\mathrm{LN}$ metastasis ( $\mathrm{rs}=0.550,0.583,0.526$, all $\mathrm{P}<0.001)$. In addition, maximum
Table 2 Radiological characteristics on MRI

\begin{tabular}{|c|c|c|c|}
\hline Characteristics & $\mathrm{LN}-(\mathrm{n}=47)$ & $\mathrm{LN}+(\mathrm{n}=23)$ & $P$ value \\
\hline MTL $(\mathrm{cm})$, mean $\pm \mathrm{SD}$ & $3.14 \pm 1.08$ & $4.21 \pm 1.07$ & $<0.001^{*}$ \\
\hline Tumor location, n (\%) & & & 0.163 \\
\hline Upper & $8(17.0)$ & $3(13.0)$ & \\
\hline Middle & $17(36.2)$ & $14(60.9)$ & \\
\hline Lower & $22(46.8)$ & $6(26.1)$ & \\
\hline CP, n (\%) & & & 0.356 \\
\hline$<1 / 4$ & 7 (14.9) & $1(4.3)$ & \\
\hline $1 / 4-1 / 2$ & $20(42.6)$ & $8(34.8)$ & \\
\hline $1 / 2-3 / 4$ & $13(27.7)$ & $11(47.8)$ & \\
\hline$>3 / 4$ & $7(14.9)$ & $3(13.0)$ & \\
\hline mrT stage, $\mathrm{n}(\%)$ & & & $<0.001^{*}$ \\
\hline $1 / 2$ & $30(63.8)$ & $2(8.7)$ & \\
\hline $3 / 4$ & $17(36.2)$ & $21(91.3)$ & \\
\hline mrEMVI, n (\%) & & & $<0.001^{*}$ \\
\hline Absent & $44(93.6)$ & $10(43.5)$ & \\
\hline Present & $3(6.4)$ & $13(56.5)$ & \\
\hline $\begin{array}{l}\text { mrLNM (morphology), } n \\
(\%)\end{array}$ & & & 0.203 \\
\hline Absent & $26(55.3)$ & $9(39.1)$ & \\
\hline Present & $21(44.7)$ & $14(60.9)$ & \\
\hline mrLNM (CSE), n (\%) & & & $<0.001^{*}$ \\
\hline Absent & $40(85.1)$ & $10(43.5)$ & \\
\hline Present & 7 (14.9) & $13(56.5)$ & \\
\hline
\end{tabular}

*, $\mathrm{P}<0.05$. MRI, magnetic resonance imaging; $\mathrm{LN}-$, pathological N0 stage; LN+, pathological N1-N2 stage; MTL, maximum tumor length; SD, standard deviation; CP, circumferential percentage; $\mathrm{mrT}$ stage, $\mathrm{T}$ stage on MRI; mrEMVI, extramural venous invasion on MRI; mrLNM, lymph node metastasis on MRI; CSE, chemical shift effect.

and kurtosis of T2 map, and maximum and variance of PD map showed significant differences between two groups ( $\mathrm{P}=0.032,0.045,0.016$, and 0.047 , respectively), and were correlated with LN metastasis ( $\mathrm{rs}=0.257,0.242,0.291$, $0.239, \mathrm{P}=0.031,0.044,0.032,0.015,0.047$, respectively).

The energy of the T1 map, T2 map, and PD map demonstrated superior predictive performance in $\mathrm{LN}$ metastasis with an AUC of 0.838 [95\% confidence interval (CI): 0.731-0.915], 0.858 (95\% CI: 0.754-0.930), 0.823 
Table 3 Histogram parameters between the LN- and LN+ groups and ROC curve results

\begin{tabular}{lccccccc}
\hline Parameters & LN- $(\mathrm{n}=47)$ & $\mathrm{LN}+(\mathrm{n}=23)$ & $\mathrm{P}$ value & AUC $(95 \% \mathrm{Cl})$ & Cutoff value & Sensitivity $(\%)$ & Specificity $(\%)$ \\
\hline Energy_T1 map $\left(\times 10^{10}\right)$ & $2.07 \pm 1.56$ & $5.06 \pm 3.34$ & $<0.001$ & $0.838(0.731-0.915)$ & $>2.61$ & 87.0 & 78.7 \\
Energy_T2 map $\left(\times 10^{7}\right)$ & $7.84 \pm 5.65$ & $19.50 \pm 10.88$ & $<0.001$ & $0.858(0.754-0.930)$ & $>10.45$ & 82.6 \\
Energy_PD map $\left(\times 10^{7}\right)$ & $3.02 \pm 2.24$ & $7.32 \pm 4.61$ & $<0.001$ & $0.823(0.714-0.904)$ & $>3.06$ & 87.0 \\
Maximum_T2 map $\left(\times 10^{2}\right)$ & $9.01 \pm 6.68$ & $13.10 \pm 7.11$ & 0.032 & $0.658(0.535-0.767)$ & $>9.01$ & 60.9 \\
Kurtosis_T2 map $\left(\times 10^{2}\right)$ & $2.38 \pm 3.56$ & $4.38 \pm 4.38$ & 0.045 & $0.648(0.525-0.759)$ & $>3.74$ & 47.8 \\
Maximum_PD map $\left(\times 10^{2}\right)$ & $1.02 \pm 0.14$ & $1.07 \pm 0.11$ & 0.016 & $0.673(0.550-0.780)$ & $>1.07$ & 80.9 & 87.0 \\
Variance_PD map $\left(\times 10^{2}\right)$ & $1.04 \pm 0.63$ & $1.13 \pm 0.34$ & 0.047 & $0.647(0.523-0.757)$ & $>0.87$ & 82.6 & 57.1 \\
\hline
\end{tabular}

$\mathrm{LN}-$, pathological NO stage; $\mathrm{LN}+$, pathological N1-N2 stage; ROC, receiver operating characteristic; AUC, area under the ROC curve; Cl, confidence interval; PD, proton density; LN, lymph node.

(95\% CI: 0.714-0.904), respectively (Figure 4D). Other parameters showed moderate diagnostic power in predicting LN metastasis.

\section{Multivariate analysis}

Only the noninvasive clinical and pathological factors, radiological features, and histogram parameters with a $P$ value $<0.05$ in the univariate analysis were included in multivariate logistic analyses. The energy of the T2 map and PD map demonstrated strong collinearity with the energy of the T1 map (VIF $=13.908$, and 12.606, respectively). It was subsequently excluded due to their similar AUCs $(\mathrm{P}=0.690$ and 0.395 in the DeLong test, respectively) and relatively lower sensitivity and specificity. The following covariates were entered into the multivariate logistic analyses, including CEA, CA199, MLT, mrT stage, mrEMVI, mrLN (CSE), the energy of T1 map, maximum of T2 map, kurtosis of T2 map, maximum of PD map, and variance of PD map. According to multivariate regression analyses, the energy of T1 map [odds ratio (OR) $=1.683,95 \%$ CI: $1.207-2.346$, $\mathrm{P}=0.002]$ and mrEMVI (OR $=10.853$, 95\% CI: 2.339 50.364, $\mathrm{P}=0.002$ ) resulted as significant predictors of $\mathrm{LN}$ metastasis. The model showed good accuracy in predicting LN status with a correct overall percentage of $85.7 \%$ and goodness of fit in the Hosmer-Lemeshow test $(\mathrm{P}=0.305)$.

\section{Diagnostic performance}

The performance of the morphological features, CSE, the energy of the T1 map, CSE + energy of T1 map, and CSE + energy of T1 map + mrEMVI in predicting LN metastasis are listed in Table 4, and their ROCs are shown in Figure 5. Better diagnostic efficiency was shown by CSE compared to morphological features, with AUCs of 0.708 and 0.581 , respectively $(\mathrm{P}=0.0015)$. Also, the energy of the T1 map (AUC $=0.838)$ significantly improved the predictive performance compared to morphological features and CSE ( $\mathrm{P}=0.0002$ and 0.0485 , respectively). The AUC of their combinations was significantly increased compared with morphological features or CSE alone, but there was no statistically significant difference between these combinations and the T1 map's energy, although the AUC was slightly improved. The results of the DeLong test are presented in Figure 6.

\section{Discussion}

This study suggested that the SyMRI-derived histogram parameters of the primary tumor could be useful for predicting regional LN metastasis in rectal cancer, and energy demonstrated superior predictive performance. Also, we found that combining the energy of the T1 map, CSE, and mrEMVI may lead to significant improvements in AUC, sensitivity, and specificity compared with morphological characteristics or CSE alone.

Reliable evaluation of metastatic LNs, which are the main indicators of unfavorable survival and distant metastasis, remains challenging. A previous study found that histogram parameters based on diffusion kurtosis imaging were associated with LN status with an AUC, sensitivity, and specificity of $0.735,0.556$, and 0.884 , respectively (10). Moreover, Liu et al. observed significant differences in histogram parameters of intravoxel incoherent motion 

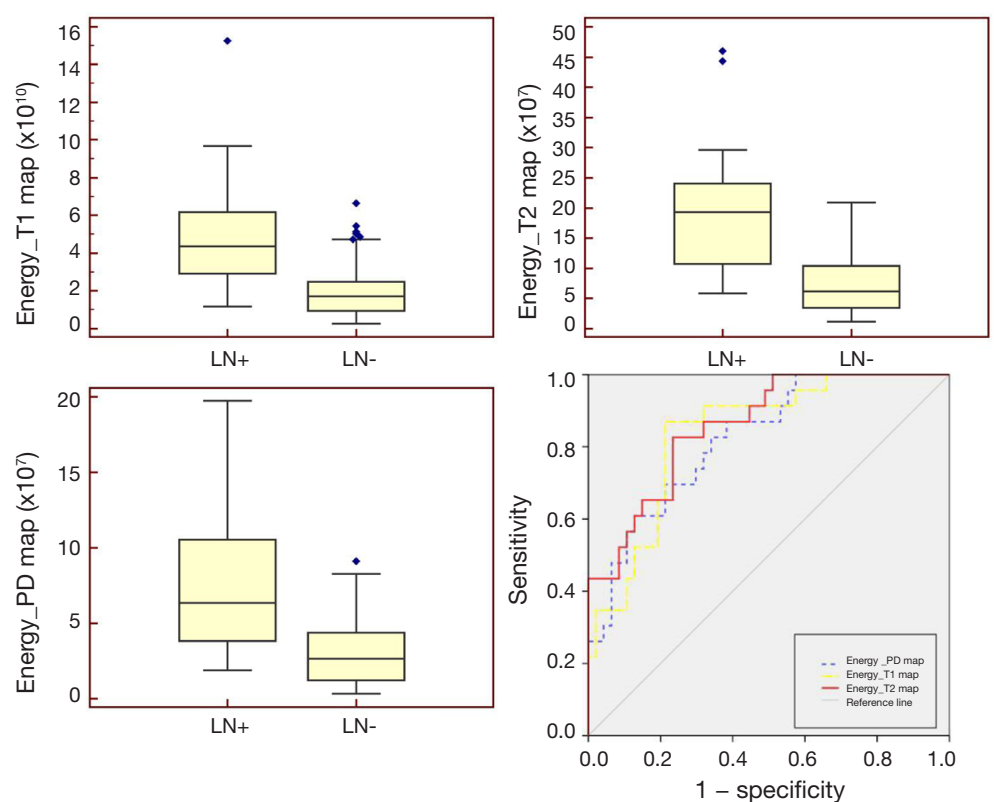

Figure 4 Box-and-whisker plots and ROC curves. (A-C) Box-and-whisker plots show the energy of the T1 map, T2 map, and PD map for the $\mathrm{LN}$-negative $(\mathrm{LN}-)$ and $\mathrm{LN}$-positive $(\mathrm{LN}+)$ groups. Significant differences were found between the two groups (all $\mathrm{P}<0.001)$; (D) the ROC curves in predicting LN metastasis with areas under the ROC curve of $0.838,0.858$, and 0.823 , respectively. PD, proton density; LN, lymph node; ROC, receiver operating characteristic.

Table 4 Predictive performance of different methods for predicting LN metastasis

\begin{tabular}{|c|c|c|c|c|c|}
\hline Methods & AUC (95\% Cl) & Sensitivity (\%) & Specificity (\%) & PPV (\%) & NPV (\%) \\
\hline mrLN (CSE) & $0.708(0.587-0.811)$ & 56.5 & 85.1 & 65.0 & 80.0 \\
\hline Energy_T1 map & $0.838(0.731-0.915)$ & 87.0 & 78.7 & 66.7 & 92.5 \\
\hline mrLN (CSE) + energy_T1 map & $0.858(0.754-0.930)$ & 91.3 & 70.2 & 60.0 & 94.3 \\
\hline
\end{tabular}

LN, lymph node; AUC, area under the receiver operating characteristic curve; Cl, confidence interval; PPV, positive predictive value; NPV, negative predictive value; mrLN, LN on MRI; CSE, chemical shift effect; mrEMVI, extramural venous invasion on MRI.

imaging between pN0 and pN1-2 patients with an AUC, sensitivity, and specificity of $0.751,0.962$, and 0.453 , respectively (25). A recent study showed that the primary tumor histogram parameters on T2WI were associated with metastatic LN with an AUC, sensitivity, and specificity of $0.750,93.5$, and 52.6, respectively (22). However, so far, no study has explored the value of histogram parameters derived from SyMRI. Our study indicated that histogram parameters of SyMRI were valuable for evaluating $\mathrm{LN}$ status with a preferable predictive performance compared with these studies (AUC $=0.838,95 \%$ CI: $0.731-0.915$, sensitivity and specificity of 0.870 and 0.787 , respectively). Furthermore, SyMRI-derived histogram parameters were found to be significantly correlated with $\mathrm{LN}$ metastasis.

We found that the T1 map, T2 map, and PD map energy achieved good performance in predicting LN status with AUCs of $0.838,0.858$, and 0.823 , respectively. Besides, the energy of the T1 map was a significant and independent predictor of LN metastasis. This suggests that energy might be a reliable predictor of regional LN metastasis in rectal cancer. The first order's energy is a measure of the magnitude of voxel values in an image, and larger values 


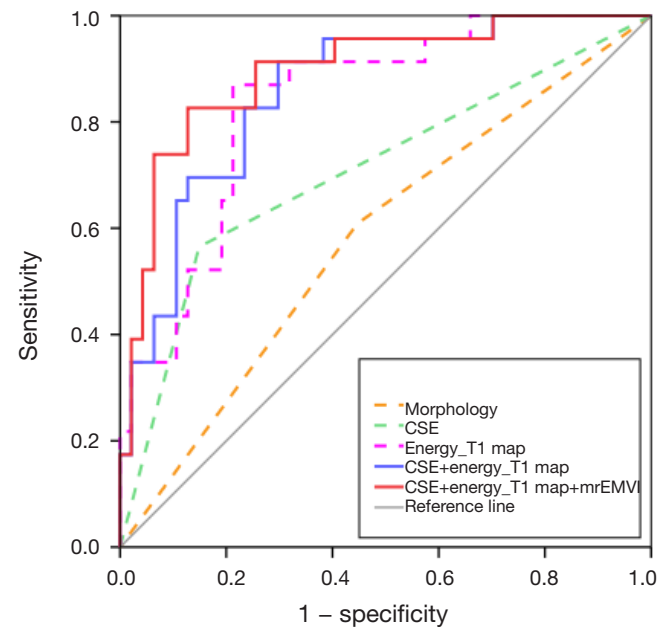

Figure 5 The ROC curves of morphological features, CSE, energy of T1 map, CSE + energy of T1 map, and CSE + energy of T1 map + mrEMVI. The combination of CSE + energy of T1 map + mrEMVI showing the highest AUC of 0.895 with a sensitivity of $82.6 \%$ and specificity of $87.2 \%$. ROC, receiver operating characteristic; CSE, chemical shift effect; mrEMVI, extramural venous invasion on magnetic resonance imaging; AUC, area under the ROC curve.

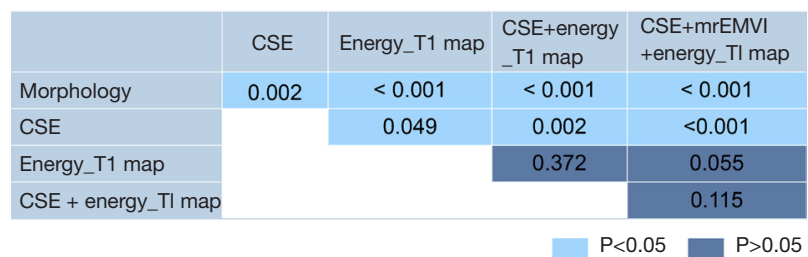

Figure 6 The difference in the predictive performance of LN metastasis between different risk factors using the DeLong test. LN, lymph node; CSE, chemical shift effect; mrEMVI, extramural venous invasion on magnetic resonance imaging.

imply a greater sum of the squares of these values (24). Antunovic et al. found that higher energy on 2-deoxy2(fluorine-18)fluoro-D-glucose $\left[\left({ }^{18} \mathrm{~F}\right) \mathrm{FDG}-\mathrm{PET} / \mathrm{CT}\right]$ represented poorer histological grade of breast cancer (26), which was consistent with our study. Conversely, Lu et al. reported that lower energy on the ADC map suggested a higher $\mathrm{T}$ stage of rectal cancer (27), but they used the energy of the gray-level co-occurrence matrix, which is a measure of heterogeneity in the image, and lower energy meant more heterogeneity. Therefore, these seemingly contradictory results are actually because the energy is from different matrices and therefore has different meanings. In the present study, we found differences in the maximum of T2 map and PD map, kurtosis (which indicates the peakedness of intensity) of T2 map, and variance of PD map between the $\mathrm{LN}-$ and $\mathrm{LN}+$ groups; however, these were not independent risk factors and had moderate predictive efficacy. This further revealed that multiple histogram parameters derived from different MR sequences played a potential role in predicting $\mathrm{LN}$ metastasis, and energy showed a superior predictive performance.

Our study's univariate analysis showed that the T stage and EMVI were associated with LN status in both pathological and MRI evaluation. Since only noninvasive features were entered in the multivariate logistic analyses, this study only explored whether the mrT stage and mrEMVI were independent risk factors for LN metastasis, while the pathological results were not included. Consequently, mrEMVI was an independent risk factor of LN metastasis, which was accordant with previous studies (28-30), whereas the mrT stage was removed at the multivariate logistic regression. In this study, $87.5 \%$ of the $\mathrm{T} 1 / 2$ cases were categorized as stage-T2, and $78.9 \%$ of T3/4 cases were assessed as T3 with an extramural depth of tumor invasion $\leq 5 \mathrm{~mm}$, as tumors with obvious extramural invasion would receive nCRT rather than direct surgery. Therefore, in the study, the impact of the primary tumor T stage was weakened. Also, we found that CEA, CA199 levels, and MTL were related to LN status but were not independent risk factors of $\mathrm{LN}$ metastasis. Whether CEA, CA199 levels, and MTL could serve as a predictor of LN metastasis remains controversial, possibly due to the heterogeneous populations of rectal cancer patients across different studies. Several studies have reported no associations between CEA, CA199 levels, MTL, and LN status (28-30), while others found that they were preoperative predictors of $\mathrm{LN}$ metastasis $(31,32)$.

Our study indicated that CSE has better performance in predicting metastatic $\mathrm{LN}$ compared to morphological features. A previous study explained CSE on the anatomical and physiological basis of the LN: between the outermost capsule and the cortex, there is a subcapsular sinus in which lymphatic fluid flows freely (33). Therefore, the subcapsular sinus and the mesorectal fat around the LN from the fat-water interface lead to the CSE along the frequency-encoding gradient (7). When tumor cells infiltrate 
the subcapsular sinus via afferent lymphatic vessels, the fat-water interface is destroyed, and CSE is interrupted, obliterated, or deformed. Moreover, the T1 map energy significantly improved the predictive performance of $\mathrm{LN}$ metastasis compared to morphological features and CSE. This suggests that histogram parameters could better reflect the potential tumor heterogeneity and aggressive biology of malignant tumors, which was consistent with previous studies $(21,22)$. Combination of the energy of the T1 map, CSE, and mrEMVI showed a superior AUC and a reliable sensitivity and specificity, thus suggesting that combining the qualitative features of LN, quantitative parameters of the primary tumor, and other characteristics related to the tumor malignancy enables a more accurate assessment of LN metastasis.

Several participants with locally advanced rectal cancer did not receive nCRT in this study, some of which were due to preoperative under-staging, and some were due to the participants' desire for direct surgery. The presence of under-staging patients may have made our results more beneficial in detecting clinically false-negative cases. The subset of patients wishing to undergo direct surgery may have somewhat affected the identification of non-metastatic and micro-metastatic LN patients, while it would further reflect the real clinical practice.

There were some limitations to this study. Firstly, the study included relatively small sample size, especially in the LN positive cohort. Secondly, a selection bias might be present due to the most suspicious $\mathrm{LN}+$ cases having received nCRT and, consequently, being excluded from the study. Thirdly, we investigated the primary tumor histogram parameters to predict the overall $\mathrm{LN}$ status and did not match the mrLN with the pathological specimen. Features of the LN itself should be explored in the future. Finally, our study only used histogram parameters with good repeatability; it would be beneficial to explore other textural and radiomic features in a future study of a large sample size.

In conclusion, our study suggested that SyMRI-derived histogram parameters of the primary tumor are associated with $\mathrm{LN}$ metastasis in rectal cancer and enable a more accurate prediction than traditional morphological features and CSE. A combination of the energy of the T1 map, CSE, and mrEMVI leads to superior predictive performance in predicting LN metastasis.

\section{Acknowledgments}

Funding: This study was funded by the National Natural
Science Foundation (81971589) and the Chinese Academy of Medical Sciences (CAMS) innovation fund for medical sciences (CIFMS) (2018-12M-AI-008).

\section{Footnote}

Conflicts of Interest: All authors have completed the ICMJE uniform disclosure form (available at http://dx.doi. org/10.21037/qims-20-659). The authors have no conflicts of interest to declare.

Ethical Statement: This study was approved by the Institutional Review Board of the Cancer Hospital, Chinese Academy of Medical Sciences (NCC2019C-040) and the informed consent was provided by all participants.

Open Access Statement: This is an Open Access article distributed in accordance with the Creative Commons Attribution-NonCommercial-NoDerivs 4.0 International License (CC BY-NC-ND 4.0), which permits the noncommercial replication and distribution of the article with the strict proviso that no changes or edits are made and the original work is properly cited (including links to both the formal publication through the relevant DOI and the license). See: https://creativecommons.org/licenses/by-nc-nd/4.0/.

\section{References}

1. Tong GJ, Zhang GY, Liu J, Zheng ZZ, Chen Y, Niu PP, $\mathrm{Xu} X \mathrm{XT}$. Comparison of the eighth version of the American Joint Committee on Cancer manual to the seventh version for colorectal cancer: a retrospective review of our data. World J Clin Oncol 2018;9:148-61.

2. Glynne-Jones R, Wyrwicz L, Tiret E, Brown G, Rodel C, Cervantes A, Arnold D. Rectal cancer: ESMO Clinical Practice Guidelines for diagnosis, treatment and followup. Ann Oncol 2018;29:iv263.

3. Beets GL, Figueiredo NF, Beets-Tan RG. Management of rectal cancer without radical resection. Annu Rev Med 2017;68:169-82.

4. Benson AB, Venook AP, Al-Hawary MM, Cederquist L, Chen YJ, Ciombor KK, Cohen S, Cooper HS, Deming D, Engstrom PF, Grem JL, Grothey A, Hochster HS, Hoffe S, Hunt S, Kamel A, Kirilcuk N, Krishnamurthi S, Messersmith WA, Meyerhardt J, Mulcahy MF, Murphy JD, Nurkin S, Saltz L, Sharma S, Shibata D, Skibber JM, Sofocleous CT, Stoffel EM, Stotsky-Himelfarb E, Willett 
CG, Wuthrick E, Gregory KM, Gurski L, FreedmanCass DA. Rectal Cancer, Version 2.2018, NCCN Clinical Practice Guidelines in Oncology. J Natl Compr Canc Netw 2018;16:874-901.

5. Beets-Tan RGH, Lambregts DMJ, Maas M, Bipat S, Barbaro B, Curvo-Semedo L, Fenlon HM, Gollub MJ, Gourtsoyianni S, Halligan S, Hoeffel C, Kim SH, Laghi A, Maier A, Rafaelsen SR, Stoker J, Taylor SA, Torkzad MR, Blomqvist L. Magnetic resonance imaging for clinical management of rectal cancer: updated recommendations from the 2016 European Society of Gastrointestinal and Abdominal Radiology (ESGAR) consensus meeting. Eur Radiol 2018;28:1465-75.

6. Li XT, Sun YS, Tang L, Cao K, Zhang XY. Evaluating local lymph node metastasis with magnetic resonance imaging, endoluminal ultrasound and computed tomography in rectal cancer: a meta-analysis. Colorectal Dis 2015;17:O129-35.

7. Zhang H, Zhang C, Zheng Z, Ye F, Liu Y, Zou S, Zhou C. Chemical shift effect predicting lymph node status in rectal cancer using high-resolution MR imaging with node-fornode matched histopathological validation. Eur Radiol 2017;27:3845-55.

8. Chen XL, Chen GW, Pu H, Yin LL, Li ZL, Song B, Li H. DWI and T2-weighted MRI volumetry in resectable rectal cancer: correlation with lymphovascular invasion and lymph node metastases. AJR Am J Roentgenol 2019. [Epub ahead of print]. doi: 10.2214/AJR.18.20564.

9. Yang X, Chen Y, Wen Z, Liu Y, Xiao X, Liang W, Yu S. Non-invasive MR assessment of the microstructure and microcirculation in regional lymph nodes for rectal cancer: a study of intravoxel incoherent motion imaging. Cancer Imaging 2019;19:70.

10. Cui Y, Yang X, Du X, Zhuo Z, Xin L, Cheng X. Wholetumour diffusion kurtosis MR imaging histogram analysis of rectal adenocarcinoma: correlation with clinical pathologic prognostic factors. Eur Radiol 2018;28:1485-94.

11. Yang X, Chen Y, Wen Z, Lu B, Shen B, Xiao X, Yu S. Role of quantitative dynamic contrast-enhanced MRI in evaluating regional lymph nodes with a short-axis diameter of less than $5 \mathrm{~mm}$ in rectal cancer. AJR Am J Roentgenol 2019;212:77-83.

12. Hagiwara A, Warntjes M, Hori M, Andica C, Nakazawa M, Kumamaru KK, Abe O, Aoki S. SyMRI of the brain: rapid quantification of relaxation rates and proton density, with synthetic MRI, automatic brain segmentation, and myelin measurement. Invest Radiol 2017;52:647-57.
13. Tanenbaum LN, Tsiouris AJ, Johnson AN, Naidich TP, DeLano MC, Melhem ER, Quarterman P, Parameswaran SX, Shankaranarayanan A, Goyen M, Field AS. Synthetic MRI for clinical neuroimaging: results of the Magnetic Resonance Image Compilation (MAGiC) Prospective, Multicenter, Multireader Trial. AJNR Am J Neuroradiol 2017;38:1103-10.

14. Arita Y, Takahara T, Yoshida S, Kwee TC, Yajima S, Ishii C, Ishii R, Okuda S, Jinzaki M, Fujii Y. Quantitative assessment of bone metastasis in prostate cancer using synthetic magnetic resonance imaging. Invest Radiol 2019;54:638-44.

15. Jung Y, Gho SM, Back SN, Ha T, Kang DK, Kim TH. The feasibility of synthetic MRI in breast cancer patients: comparison of T2 relaxation time with multiecho spin echo T2 mapping method. Br J Radiol 2018;92:20180479.

16. Kumar NM, Fritz B, Stern SE, Warntjes J, Lisa CY, Fritz J. Synthetic MRI of the knee: phantom validation and comparison with conventional MRI. Radiology 2018;289:465-77.

17. Mai J, Abubrig M, Lehmann T, Hilbert T, Weiland E, Grimm MO, Teichgraber U, Franiel T. T2 mapping in prostate cancer. Invest Radiol 2019;54:146-52.

18. Liu L, Yin B, Geng DY, Lu YP, Peng WJ. Changes of T2 relaxation time from neoadjuvant chemotherapy in breast cancer lesions. Iran J Radiol 2016;13:e24014.

19. Gourtsoyianni S, Doumou G, Prezzi D, Taylor B, Stirling JJ, Taylor NJ, Siddique M, Cook G, Glynne-Jones R, Goh V. Primary rectal cancer: repeatability of global and local-regional MR imaging texture features. Radiology 2017;284:552-61.

20. De Cecco CN, Ganeshan B, Ciolina M, Rengo M, Meinel FG, Musio D, De Felice F, Raffetto N, Tombolini V, Laghi A. Texture analysis as imaging biomarker of tumoral response to neoadjuvant chemoradiotherapy in rectal cancer patients studied with 3-T magnetic resonance. Invest Radiol 2015;50:239-45.

21. Jalil O, Afaq A, Ganeshan B, Patel UB, Boone D, Endozo R, Groves A, Sizer B, Arulampalam T. Magnetic resonance based texture parameters as potential imaging biomarkers for predicting long-term survival in locally advanced rectal cancer treated by chemoradiotherapy. Colorectal Dis 2017;19:349-62.

22. Yang L, Liu D, Fang X, Wang Z, Xing Y, Ma L, Wu B. Rectal cancer: can T2WI histogram of the primary tumor help predict the existence of lymph node metastasis? Eur Radiol 2019;29:6469-76.

23. Peng Y, Tang H, Meng X, Shen Y, Hu D, Kamel I, Li 
Z. Histological grades of rectal cancer: whole-volume histogram analysis of apparent diffusion coefficient based on reduced field-of-view diffusion-weighted imaging. Quant Imaging Med Surg 2020;10:243-56.

24. van Griethuysen JJM, Fedorov A, Parmar C, Hosny A, Aucoin N, Narayan V, Beets-Tan RGH, Fillion-Robin JC, Pieper S, Aerts HJWL. Computational radiomics system to decode the radiographic phenotype. Cancer Res 2017;77:e104-7.

25. Liu L, Liu Y, Xu L, Li Z, Lv H, Dong N, Li W, Yang Z, Wang Z, Jin E. Application of texture analysis based on apparent diffusion coefficient maps in discriminating different stages of rectal cancer. J Magn Reson Imaging 2017;45:1798-808.

26. Antunovic L, Gallivanone F, Sollini M, Sagona A, Invento A, Manfrinato G, Kirienko M, Tinterri C, Chiti A, Castiglioni I. [(18)F]FDG PET/CT features for the molecular characterization of primary breast tumors. Eur J Nucl Med Mol Imaging 2017;44:1945-54.

27. Lu Z, Wang L, Xia K, Jiang H, Weng X, Jiang J, Wu M. Prediction of clinical pathologic prognostic factors for rectal adenocarcinoma: volumetric texture analysis based on apparent diffusion coefficient maps. J Med Syst 2019;43:331.

28. Chen LD, Liang JY, Wu H, Wang Z, Li SR, Li W, Zhang XH, Chen JH, Ye JN, Li X, Xie XY, Lu MD, Kuang M, Xu JB, Wang W. Multiparametric radiomics

Cite this article as: Zhao L, Liang M, Shi Z, Xie L, Zhang H, Zhao X. Preoperative volumetric synthetic magnetic resonance imaging of the primary tumor for a more accurate prediction of lymph node metastasis in rectal cancer. Quant Imaging Med Surg 2021;11(5):1805-1816. doi: 10.21037/qims-20-659 improve prediction of lymph node metastasis of rectal cancer compared with conventional radiomics. Life Sci 2018;208:55-63.

29. Pomerri F, Crimi F, Veronese N, Perin A, Lacognata C, Bergamo F, Boso C, Maretto I. Prediction of N0 irradiated rectal cancer comparing MRI before and after preoperative chemoradiotherapy. Dis Colon Rectum 2017;60:1184-91.

30. Zhang XY, Wang S, Li XT, Wang YP, Shi YJ, Wang L, Wu AW, Sun YS. MRI of extramural venous invasion in locally advanced rectal cancer: relationship to tumor recurrence and overall survival. Radiology 2018;289:677-85.

31. Zhou C, Liu HS, Liu XH, Zheng XB, Hu T, Liang ZX, He XW, He XS, Hu JC, Wu XJ, Wu XR, Lan P. Preoperative assessment of lymph node metastasis in clinically node-negative rectal cancer patients based on a nomogram consisting of five clinical factors. Ann Transl Med 2019;7:543.

32. Miki H, Akiyoshi T, Ogura A, Nagasaki T, Konishi T, Fujimoto Y, Nagayama S, Noma H, Saiura A, Fukunaga Y, Ueno M. Pretreatment serum carbohydrate antigen 19-9 concentration is a predictor of survival of patients who have undergone curative resection of stage IV rectal cancer. Dig Surg 2018;35:389-96.

33. Ohtani O, Ohtani Y. Structure and function of rat lymph nodes. Arch Histol Cytol 2008;71:69-76. 